\title{
Research on the Beef Product Traceability System based on the Internet of Things Technologies
}

\author{
Jian Zhao ${ }^{1, a}$, Longju $\mathrm{Wu}^{1, \mathrm{~b}}$, Zhe Wang ${ }^{1, \mathrm{c}}$, \\ Yan Peng ${ }^{1}$, Yanmin $\mathrm{Li}^{1}$ and Chao Zhang ${ }^{1}$ \\ ${ }^{1}$ College of Computer Science and Technology, Jilin University, Changchun, 130012, China \\ ajzhao10@mails.jlu.edu.cn, ${ }^{\mathrm{b}} \mathrm{ljwu10@mails.jlu.edu.cn,} \mathrm{'wz2000@jlu.edu.cn}$
}

Keywords: Internet of things, RFID, beef product traceability system

\begin{abstract}
With our concern about food safety issues, many areas have adopted the food traceability system in management and monitoring the entire chain of food production, processing and marketing. There are still many issues to the existing food traceability system of China such as inconsistent traceability information flow, the non-standard content and the difficulty of sharing and exchanging data. This paper studies and designs beef product traceability system by the introduction of the Internet of things technologies and combining with the technology of RFID and EPC standard. The beef product traceability system that has important theoretical significance and practical value can provide consumers with beef product information query and facilitate the logistics responsibility traceability for managers.
\end{abstract}

\section{Introduction}

With the continuous development of scientific and technological level and the continuous improvement of people's health awareness, the issue related to food hygiene and safety is becoming one of the focuses of widespread concern. The food safety traceability of China began in 2003 . The retrospective codes that are non-standardized and non-compatible do not use the Global Identification System and form information "islands". The information "islands" increase the computer system running cost and cause the delay and uncertainty of supply chain and the waste of time and resources. Moreover it is impossible for regulators to ensure the food safety to provide effective basis by the information "islands". Establishing the food safety traceability can achieve directional recall in the event of food safety incidents and strengthen the government supervision on food safety and production enterprises' good faith consciousness so that it promotes agricultural industrialization development. There are still many issues to the existing food traceability system of China such as inconsistent traceability information flow, the non-standard content and the difficulty of sharing and exchanging data. This paper studies and designs beef product traceability system by the introduction of the Internet of things technologies and combining with the technology of RFID and EPC standard. The beef product traceability system effectively ensures the quality of the beef from the beef cattle breeding, processing including slaughtering and processing, transportation, marketing and other aspects.

\section{The Conceptions of Internet of Things Technologies}

The Conception of Internet of Things. Internet of Things technologies, which began in the Massachusetts Institute of Technology in 1999, refers to the concept of expanding the traditional Internet into the information exchange network between the object and the object of the real world. Initially, the Internet of Things is only based on RFID technology and achieves the exchange and sharing of information through the information stored in the label, as well as wireless network media [1]. At present, the of the Internet of things, whose coverage includes not only RFID, sensing devices but also laser scanner, GPS and infrared sensors, follows the standard protocol and connects to any article and supports information exchange and communication between the object and the object [2]. 
The core technologies of Internet of Things concept include two aspects [3]: one is the RFID radio frequency identification technology that uses RF signals to identify the target and collected information; the other is Electronic Product Code technology that implements the global items of information sharing by assigning a unique code to each object and depositing the code in the silicon chip made of electronic tags.

EPC and RFID Technologies. The EPC, standing for "electronic product code", is the unique identifier of each entity object, which is composed of a set of numbers by a version number and the other three sections of data (domain name manager, object classification, serial number). The EPC solves the identification and tracking of individual commodities and establish a global identity standard for each commodity.

The RFID, standing for "radio frequency identification", is a radio frequency identification technology to identify the target object and access to relevant information technology. The RFID that is a non-contact automatic identification technology works without human intervention and can work in a variety of harsh environments by identifying the target object automatically and accessing to relevant data [4].

\section{The Design of Beef Product Traceability System based on Internet of Things Technology}

System Design. From the actual process of starting beef products in logistics, the beef product traceability system based on Internet of things technology consists of three parts that are beef products circulation monitoring subsystem, beef product information query system and beef products Internet data center. The overall frame is shown in Fig. 1.

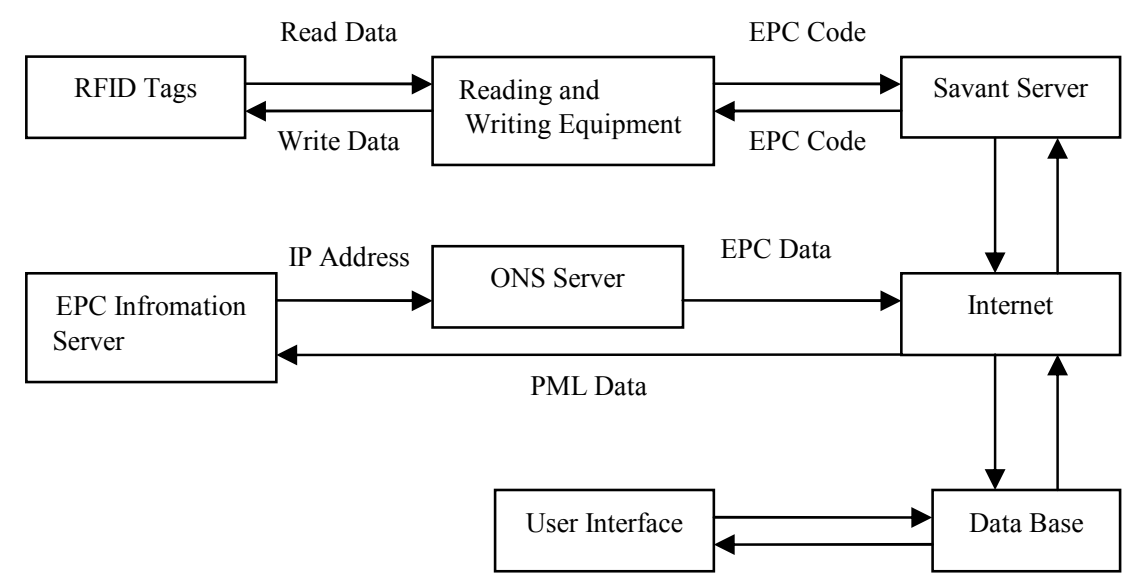

Beef products production and circulation monitoring

Beef product the internet of things data center

Beef product information query subsystem

Fig. 1. The overall frame of the system

Beef products circulation monitoring subsystem is responsible for the monitoring of the production, processing, transportation and sales of beef products. Within a certain time after the birth of each head of cattle, the farm staff will install electronic tag that has a unique number on the ear of each cow so that each head of cattle will be established electronic identity card. Through the EPC radio frequency identification system and Savant system, the information of the beef products in the logistics distribution system can be accessed to and EPC encoded data can be transmitted to the Savant system [5]. According to this information, the Savant system can track beef product and Network quality and liability traceability management can be achieved.

As the core part of the system, beef products Internet data center provides for the whole system of powerful database support and service. The data information collected by the other modules in the system will be summarized in the center database, the center saves and records the various information of the food in every aspect of production and analyzes and summarizes the information collected and provides technical services and information support for managers. The center can transmit the information from the data collection layer to the Internet through wireless or wired communication mode including The Internet network, GPR network, GSM and CDMA [1]. 
Beef product information query system provides the information of beef products circulation monitoring subsystem. The users can query the safety of beef product through online information platform and the information contains detailed information in each link including the production, processing, transportation and sales of beef products.

RFID Tags. As the data carrier of beef products radio frequency identification system, RFID tags can achieve automatic recognition of beef products by the radio frequency signal and obtain the related data of the beef quality and variety of beef products.

Read-write Device. Read-write device is used to read information and data of beef products and save the information and data in the electronic tag information. RFID tags send electromagnetic waves to the read-write device and Read-write device decode the electromagnetic waves to the data of beef products.

EPC Code. EPC code can save the embedded information of beef products including the quality, validity, place of origin, and responsible person by the standard structure of the 96 encoding. With the circulation of beef products, the embedded information can be synchronized in order to continue to identify and query [6].

Savant Server. The RFID tags of beef products in logistics and sales process will produce a series of EPC numbers [7]. Savant server, which is used to send and manage EPC data, can be installed in the beef product market or logistics and distribution centers and other places to do data proofreading, data transmission and storage management.

ONS Server. The function of ONS server is to parse the domain name, which means that matching the appropriate product information and EPC numbers in order to determine the reference information of the beef products. Savant server can obtain the address of the product server through the ONS server.

Under the common action of these modules, information flow of beef products is as follows: Read-write device scans the RFID tags of the beef products to pass information to the Savant server; the Savant server processes the information and transmit the information to EPC Information Server combined with the ONS server; the client software system access to information of beef products by ONS and obtain the services of beef product quality and traceability [8].

RFID. The key of Internet of things technologies is to achieve automatic identification and sharing of information of the object's position and status by RFID, GPS and WLAN. The system uses the read-write device with GPS, network functions and programmable features, including mobile and fixed. Fixed read-write devices are placed into the farms, warehouses, supermarkets and other fixed place for checking the status of products. RFID-tagged products through the channel can be automatically read the corresponding data and RFID tag will be write variety of products, specifications, origin, batch, location, work unit, time and other information, at the same time correlation information will be passed to the enterprise internal management systems and networking data center without the need for additional staff to assist or control. Mobile read-write device, which is light weight and small size and can be carried by the staff, is mainly for employees and food inspection workers and can perceive varieties, specifications, origin, batch, status and other information of food within the detection range. Mobile devices can also pass the RFID information of beef products to the Internet of Things through wireless means. With the process of farming, storage, processing and transportation, read-write device can write the information to the corresponding electronic tag through the collection of ambient temperature, temperature, light and other information with the associated sensors. Connecting beef products to the Internet of Things can achieve the purpose of the regulation on beef products by the positioning function read-write devices.

EPC Information. The RFID tags of the beef product traceability system consist of the chip and antenna, and each RFID tag has a unique EPC code. EPC coding has the advantage of safety and uniqueness. The encoded content can be stored beef product category, place of origin, processing enterprises, date of production and logistics status information. Current EPC code median of three 
kinds that are 64, 96 and 256, as shown in Table 1 Considering the information types and amount of beef products logistics and taking into account the balance of costs and complete information, The encoding digits of the design are 96 [9].

Table 1. EPC Coding Structure

\begin{tabular}{|c|c|c|c|c|c|}
\hline $\begin{array}{c}\text { Encoding } \\
\text { Scheme }\end{array}$ & Type & Version & $\begin{array}{c}\text { Domain } \\
\text { Management }\end{array}$ & $\begin{array}{c}\text { Object } \\
\text { Classification }\end{array}$ & Serial Number \\
\hline \multirow{3}{*}{ EPC-64 } & Type I & 2 & 21 & 17 & 24 \\
\cline { 2 - 6 } & Type II & 2 & 15 & 13 & 34 \\
\cline { 2 - 6 } & Type III & 2 & 26 & 13 & 23 \\
\hline \multirow{2}{*}{ EPC-96 } & Type I & 8 & 28 & 24 & 36 \\
\hline \multirow{2}{*}{ EPC-256 } & Type I & 8 & 32 & 56 & 160 \\
\cline { 2 - 6 } & Type II & 8 & 64 & 56 & 128 \\
\cline { 2 - 6 } & Type III & 8 & 128 & 56 & 64 \\
\hline
\end{tabular}

Logistics Information of beef products fall into two categories, namely fixed information and variable information. The fixed information stores the basic properties of beef products, including beef, product name, product price and product place of origin. The variable information changes with specific varieties of beef products and logistics location, including product shelf life and product testing number.

The variable information is real-time refresh in accordance with standard with the changing of the place of origin, processing location and the liquidity of the market of beef products. EPC code is stored in the electronic label of beef products to be identified in order to be systems read, store and query and build the product quality and traceability system.

When beef products are in a production or logistics process, RFID tags with EPC-encoded can be used as the unique identifier of the product. Do positioning tracing on beef product in each logistics links with the EPC code of beef product. Deploy and configure the Savant system in the critical current nodes. When the beef products are in the process of storage, transport and packaging, the information reader will read and store electronic code information of products in the effective distance.

Savant. As the standard program module integrated device, Savant consists of three standard modules that are Event Management System, Real-time In-Memory Event Database and Task Management System. In this paper, the Java platform environment is chosen for the design and development and SQL Server is used for the background database with the actual demand for the flow of beef products. The system uses the GT4 (Globus Tookit 4) platform and uses grid technology based on SOA (Service Oriental Architecture) thinking to design distributed Savant. Globus is an open architecture and protocols and services it provides provide a favorable technical support for the design of Savant and facilitate the integration, maintenance and upgrades of system resources. The implementation of the Savant system can take full advantage of many services resources the GT4 provides, manage effectively internal resources of system and coordinate the interaction with external systems so that the Savant system of beef product traceability system can be in coordination, reliable operation [10].

On this basis, Savant exist in the tree structure in the entire back system, shown in Fig. 2 Savant of beef traceability system are distributed in the place of origin, warehouse, transport, and regional centers and distribution centers for beef product data capture, monitoring and delivery. In Fig. 2, ES is edge Savant node and in the leaf position in the tree structure. The function of these nodes are to connect to the RFID information collection equipment and acquire and store data real-timely, at the same time transmit data to the IS node. IS nodes are internal nodes of the tree structure, which are non-leaf nodes and the parents nodes of ES codes. The main functions of IS nodes are to bring together beef products information and data of the ES nodes to prepare for the EPC information queries. Each time accessing to product information, EPC Savant will store EPC codes and code 
information of read-write devices. Another function of the IS nodes is statistics EPC data and doing the processing and integration of EPC data. Therefore, IS nodes of Savant are usually located in data centers or beef products regional centers of quality traceability information system.

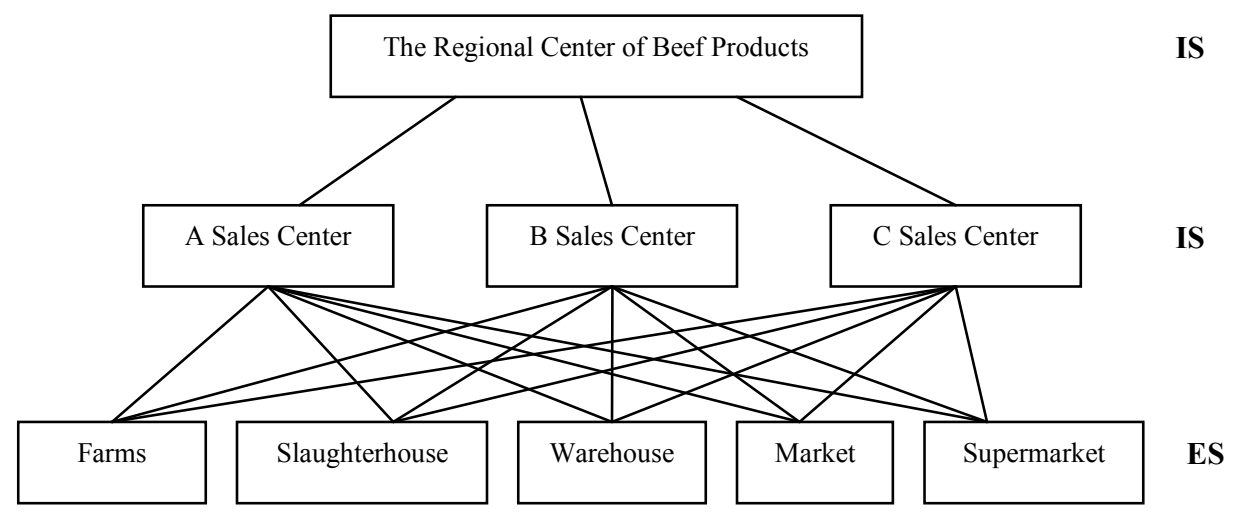

Fig. 2. The tree structure of Savant

\section{Summary}

This article designs the beef product traceability system based on the introduction of the Internet of Things technology and combined with RFID technology and EPC standards. The findings not only provide consumers with beef products information inquiry, as well as facilitate logistics responsibility traceability for the managers, with important theoretical and practical value.

\section{Acknowledgements}

This work was supported by the National Natural Science Foundation of China (NSFC) under Grant No. 61175023, National High-Tech Program (2009AA02Z307), project of science and technology innovation platform of computing and software science (985 engineering), Project Research of Clustering Algorithm Aim at the Data with Multidimensional Mixed Attributes under Grant No. 20121102 Supported by Graduate Innovation Fund of Jilin University, Fundamental Research Funds for the Central Universities (Grant No. 201103195) and the Key Laboratory for Symbolic Computation and Knowledge Engineering, Ministry of Education, China.

\section{References}

[1] YangXiao, Xuemin Shen, Bo Sun, Security and privacy in RFID and applications in Telemedicine, IEEE Communications Magazine, 14 (2010) 23-24.

[2] Feldhofer M, Dominikus S, Wolkerstorfer J, Strong authentication for RFID systems using the AES algorithm, Workshop on Cryptographic Hardware and Embedded Systems, CHES2010, LNCS, Springer, (2010) 38-40.

[3] Ying Liu, RFID Technical Principle and Its Application, Journal of the Central University for Nationalities (Natural Sciences Edition), 15 (2006) 66-67.

[4] Zhongwei Luo, Yunpeng Xing, Exploring the Development Model of the Internet of Things Industry_ C Case analysis based on Chinaexpress, Macroeconomics, 12 (2010) 24-29.

[5] Melanie R Rieback, Bruno Crispo, Andrew S Tanenbaum, The evolution of RFID Security, RFID Technology, 9 (2006) 747-749.

[6] Zhongmin Wang, EPC and The Internet of Things, China Standard Press, Beijing, 2009. 
[7] Songsen Yu, Zhiping Wang, Yiju Zhan, A New "Internet of Things" Model Based on EPC, Guangdong Automation \& Information Engineering, 3 (2010) 10-12.

[8] Rui Chen, IOT:A Strategic Opportunity for National Innovative Development in Post IP Era, Bulletin of Chinese Academy of Sciences, 1 (2010) 53-54.

[9] Chao Ai, Huaming Fu, The Internet of Things Design Based on RFID Technology in Modern Factory, Electronic Component \& Device Applications, 9 (2011) 75-77.

[10]Honghui He, Guoqing Liu, Botao Liu, Numerical Simulation of Upward Gas-Liquid Two-Phase Flow in Vertical Tube, Spacecraft Environment Engineering, 21 (2004) 41-47. 